\title{
Massive gravity on a brane
}

\author{
Z. Chacko, ${ }^{1, *}$ M. L. Graesser, ${ }^{2, \dagger}$ C. Grojean, ${ }^{3,4, \ddagger}$ and L. Pilo ${ }^{5, \S}$ \\ ${ }^{1}$ Department of Physics, University of California at Berkeley, Berkeley, California 94720, USA \\ and Lawrence Berkeley National Laboratory, Berkeley, California 94720, USA \\ ${ }^{2}$ California Institute of Technology, 452-48, Pasadena, California 91125, USA \\ ${ }^{3}$ Service de Physique Théorique, CEA Saclay, F91191 Gif-sur-Yvette, France \\ ${ }^{4}$ Michigan Center for Theoretical Physics, Ann Arbor, Michigan 48109, USA \\ ${ }^{5}$ Dipartimento di Fisica 'G. Galilei,' Università di Padova, INFN, Sezione di Padova, Via Marzolo 8, I-35131 Padua, Italy
}

(Received 17 February 2004; revised manuscript received 6 July 2004; published 15 October 2004)

At present no theory of a massive graviton is known that is consistent with experiments at both long and short distances. The problem is that consistency with long distance experiments requires the graviton mass to be very small. Such a small graviton mass however implies an ultraviolet cutoff for the theory at length scales far larger than the millimeter scale at which gravity has already been measured. In this paper we attempt to construct a model which avoids this problem. We consider a brane world setup in warped anti- de Sitter spacetime and we investigate the consequences of writing a mass term for the graviton on an infrared brane where the local cutoff is of order a large (galactic) distance scale. The advantage of this setup is that the low cutoff for physics on the infrared brane does not significantly affect the predictivity of the theory for observers localized on the ultraviolet brane. For such observers the predictions of this theory agree with general relativity at distances smaller than the infrared scale but go over to those of a theory of massive gravity at longer distances. A careful analysis of the graviton two-point function, however, reveals the presence of a ghost in the low energy spectrum. A mode decomposition of the higher dimensional theory reveals that the ghost corresponds to the radion field. We also investigate the theory with a brane-localized mass for the graviton on the ultraviolet brane, and show that the physics of this case is similar to that of a conventional four dimensional theory with a massive graviton, but with one important difference: when the infrared brane decouples and the wouldbe massive graviton gets heavier than the regular Kaluza-Klein modes, it becomes unstable and it has a finite width to decay off the brane into the continuum of Kaluza-Klein states.

DOI: 10.1103/PhysRevD.70.084028

\section{INTRODUCTION}

Recently there has been considerable interest in theories of gravitation which deviate from Einstein's gravity at very long distances (for example, [1-5]). However there has been no consistent theory yet proposed which is consistent with all observations at both macroscopic and microscopic length scales [6-11]. Conceptually perhaps the simplest modification is a mass term for the graviton [12]. However this theory suffers from three difficulties which are typical of theories of massive gravity as a whole. First, unless the mass term has the FierzPauli (FP) form the theory has a ghost $[12,13]$. Second, for a graviton mass $m_{g}$ this theory has a cutoff $\left(m_{g}^{4} M_{4}\right)^{1 / 5}$ [7], where $M_{4}$ is the Planck scale. This cutoff is much too low for the theory to be simultaneously consistent with experiments at microscopic and macroscopic scales. Third, even for arbitrarily small graviton mass the longitudinal component of the massive graviton does not decouple from sources. This fact, which was first observed by van Dam, Veltman and Zakharov [13], implies that the tensor structure of the gravitational interaction deviates

\footnotetext{
*Electronic address: zchacko@thsrv.lbl.gov

${ }^{\dagger}$ Electronic address: graesser@theory.caltech.edu

${ }^{\ddagger}$ Electronic address: grojean@spht.saclay.cea.fr

${ }^{\S}$ Electronic address: pilo@pd.infn.it
}

PACS numbers: 04.50.+h

from that of Einstein gravity. While there are indications that suitable ultraviolet completions may be free of the latter problem [14-17] to date no completely satisfactory candidate theories are known [8-11].

In the absence of a known Higgs mechanism for gravity it might seem that these problems pose an insurmountable obstacle in constructing any experimentally viable theory of a massive graviton. However a closer examination suggests that this need not be the case. Consider the five dimensional brane model of Randall and Sundrum (RS) [18]. This is a simple example of a theory where the local cutoff varies from point to point in the higher dimensional space. In particular in the far infrared the cutoff of the theory is below the millimeter scale, where gravity has been measured in the laboratory. This low cutoff is completely consistent with these experiments because physics measurements on the brane at any four momentum scale $p$ are exponentially insensitive to points in the bulk where the local cutoff is lower than the scale p.

The success of this theory suggests a means whereby the problems normally associated with theories of massive gravity can be avoided. To the single brane model of Randall and Sundrum we add a second brane deep in the infrared such that the compactification radius, which is the inverse mass of the lightest Kaluza-Klein (KK) state, is of order galactic size. On this second brane we add a 
brane-localized mass term for the graviton. The extra dimension just corresponds to an interval with two boundaries (see Ref. [19] for similar constructions for gauge theories and their application for the problem of electroweak symmetry breaking). Since two-point correlators with external legs on the ultraviolet brane are exponentially insensitive to physics on the infrared brane for four momenta above the compactification scale we expect that conventional Einstein gravity will be reproduced on the ultraviolet brane at distances shorter than the compactification scale. However, at distances longer than the compactification scale, the theory is sensitive to infrared physics, with the consequence that below this scale the theory with a Fierz-Pauli mass term is expected to resemble the four dimensional Fierz-Pauli theory of a massive graviton. This then would be a concrete realization of a an experimentally viable theory of massive gravity. In this paper we investigate this proposal in detail. This picture is correct, at the price of a serious drawback though: while the theory does indeed reproduce Einstein gravity at subgalactic length scales the low energy spectrum in the four dimensional effective theory contains, in addition to a massive graviton, a ghost state. A mode decomposition of the higher dimensional theory reveals that it is the radion field which is a ghost. ${ }^{1} \mathrm{We}$ also find that this conclusion is rather general: even allowing for a non-Fierz-Pauli mass term on the IR brane the radion field is always a ghost. The analysis of the more general case is provided in Appendix B.

In the following sections we explore in detail the model we are investigating. We compute the graviton two-point function with external legs on the ultraviolet brane and show that while the predictions of the theory agree with those of general relativity for observers on the ultraviolet brane probing distance scales shorter than the compactification radius, the light states consist of a massive graviton and a ghost. We then perform a mode decomposition of the linearized theory for both the transverse traceless modes and the radion. This reveals that the transverse traceless modes of the theory without a mass term smoothly go over to the transverse traceless modes of the theory with a mass term as the mass term is turned on. However the same is not true of the radion. Instead, the radion changes discontinuously into a ghost as soon as the mass term is turned on. We also study the theory with a mass term on the ultraviolet brane and show that the predictions of this theory for observers on the ultraviolet brane agree with those of a four dimensional theory with a massive graviton. However there is one important difference: if the extra dimension is sufficiently large that the would-be graviton is heavier than the lightest regular

\footnotetext{
${ }^{1}$ Under certain circumstances theories with ghosts may in fact be viable [20,21]. However we do not pursue this possibility here.
}

Kaluza-Klein states, then it becomes unstable and it has a finite though small width to decay into the regular Kaluza-Klein states.

\section{BRANE-LOCALIZED FIERZ-PAULI MASS TERM}

\section{A. Bulk equations of motion}

We consider a brane world model whose dynamics is governed by the following action: ${ }^{2}$

$$
\begin{aligned}
S= & \int d^{5} x \sqrt{|g|}\left[\frac{\mathcal{R}}{2 \kappa_{5}^{2}}-\Lambda+\left(-\sigma_{i}+\mathcal{L}_{i}\right)\right. \\
& \left.\times \delta\left[\sqrt{g_{55}}\left(z-z_{i}\right)\right]\right],
\end{aligned}
$$

$z_{i=1,2}$ are the locations of the two branes, $\Lambda$ is the bulk cosmological constant, $\kappa_{5}$ is related to the 5D Planck (fundamental) scale $M_{5}$ by $M_{5}^{3}=1 / 2 \kappa_{5}^{2}, \sigma_{i}$ are the brane cosmological constants (tensions), and $\mathcal{L}_{i}$ are Lagrangian densities describing some boundary localized matter fields. We will fine-tune the bulk and brane cosmological constants such that the background geometry corresponds to the well-known Randall-Sundrum solution:

$$
d s^{2}=\left(\frac{R}{z}\right)^{2}\left(\eta_{\mu \nu} d x^{\mu} d x^{\nu}+d z^{2}\right)
$$

with $R^{-1}=\sqrt{-\kappa_{5}^{2} \bar{\Lambda} / 6}, \quad \sigma_{U V}=6 /\left(\kappa_{5}^{2} R\right)$ and $\sigma_{I R}=$ $-\sigma_{U V}$. The location of the branes are such that the warp factor $R / z$ is set to 1 on the ultraviolet (UV) brane $\left(z_{1}=R\right)$, and it is exponentially smaller on the IR brane $\left(z_{2}=R^{\prime} \gg R\right)$.

The aim of this paper is to study the spectrum of the physical excitations when nontrivial gravitational interactions are introduced on the branes. We thus need to consider gravitational fluctuations around the RS background solution:

$$
d s^{2}=e^{2 A}\left(\eta_{M N}+h_{M N}\right) d x^{M} d x^{N}, \quad \text { with } A=-\ln (z / R) \text {. }
$$

In the bulk, the Einstein's equations are of course independent of brane interaction terms. At the linear level and in absence of any matter beside the bulk cosmological constant, these equations read

$$
E_{M N}^{(1)} \equiv G_{M N}^{(1)}+\kappa_{5}^{2} \Lambda e^{2 A} h_{M N}=0,
$$

where $G_{M N}^{(1)}$ is the linear piece of the Einstein tensor. Using the Einstein equations of the background solution, we finally arrive at

\footnotetext{
${ }^{2}$ Our conventions correspond to a mostly plus signature $(-+$ $\ldots+)$ and the definition of the curvature is such that a Euclidean sphere has a positive curvature. Bulk coordinates will be denoted by capital Latin indices and brane coordinates by Greek indices.
} 


$$
\begin{aligned}
E_{\mu \nu}^{(1)}= & \frac{1}{2}\left(\partial_{\mu} \partial^{\sigma} h_{\nu \sigma}+\partial_{\nu} \partial^{\sigma} h_{\mu \sigma}-\square h_{\mu \nu}-\partial_{\mu} \partial_{\nu} h\right)-\frac{1}{2}\left(\partial_{\sigma} \partial_{\rho} h^{\sigma \rho}-\square h\right) \eta_{\mu \nu}-\frac{1}{2}\left(h_{\mu \nu}^{\prime \prime}-h^{\prime \prime} \eta_{\mu \nu}\right)-\frac{3}{2}\left(h_{\mu \nu}^{\prime}-h^{\prime} \eta_{\mu \nu}\right) A^{\prime} \\
& -\frac{1}{2} \partial_{\mu} \partial_{\nu} h_{55}+\frac{1}{2}\left(\square h_{55}-3 A^{\prime \prime} h_{55}-3 A^{\prime} h_{55}^{\prime}-9 A^{\prime 2} h_{55}\right) \eta_{\mu \nu}+\frac{1}{2}\left(\partial_{\mu} h_{\nu 5}^{\prime}+\partial_{\nu} h_{\mu 5}^{\prime}\right)-\partial^{\sigma} h_{\sigma 5}^{\prime} \eta_{\mu \nu}+\frac{3}{2} A^{\prime}\left(\partial_{\mu} h_{\nu 5}\right. \\
& \left.+\partial_{\nu} h_{\mu 5}\right)-3 A^{\prime} \partial^{\sigma} h_{\sigma 5} \eta_{\mu \nu},
\end{aligned}
$$

$$
\begin{aligned}
E_{\mu 5}^{(1)}= & \frac{1}{2}\left(\partial^{\sigma} h_{\sigma \mu}-\partial_{\mu} h\right)^{\prime}+\frac{3}{2} A^{\prime} \partial_{\mu} h_{55}+\frac{1}{2} \partial_{\mu} \partial^{\sigma} h_{\sigma 5} \\
& -\frac{1}{2} \square h_{\mu 5}+3 A^{\prime \prime} h_{\mu 5}-3 A^{\prime 2} h_{\mu 5}, \\
E_{55}^{(1)}= & -\frac{1}{2}\left(\partial^{\sigma} \partial^{\rho} h_{\sigma \rho}-\square h\right)+\frac{3}{2} A^{\prime} h^{\prime}-6 A^{\prime 2} h_{55} \\
& -3 A^{\prime} \partial^{\sigma} h_{\sigma 5},
\end{aligned}
$$

with the following conventions: 4D indices are raised and lowered using the flat Minkowski metric, $h$ is the 4D trace $h_{\mu}^{\mu}, \square=\partial^{\sigma} \partial_{\sigma}$ and a prime denotes a derivative with respect to the $z$ coordinate.

The bulk equations are obviously covariant under an infinitesimal general coordinate transformation that reads at the linear order:

$$
\begin{gathered}
\delta x^{M}=\xi^{M} \\
\delta h_{\mu \nu}=-\partial_{\mu} \xi_{\nu}-\partial_{\nu} \xi_{\mu}-2 A^{\prime} \xi^{5} \eta_{\mu \nu} \\
\delta h_{\mu 5}=-\xi_{\mu}^{\prime}-\partial_{\mu} \xi^{5}, \quad \delta h_{55}=-2\left(\xi^{5} e^{A}\right)^{\prime} e^{-A} .
\end{gathered}
$$

Clearly, this reparametrization invariance allows to restrict ourselves to generalized Gaussian normal (GGN) systems of coordinates:

$$
\begin{aligned}
h_{55} & =h_{\mu 5}=0, \quad \text { brane embeddings : } z=f_{i}(x), \\
i & =1,2 .
\end{aligned}
$$

Within these generalized Gaussian normal gauges, there is still a residual reparametrization invariance involving arbitrary functions, $\zeta$ and $\zeta^{\mu}$, of the 4D coordinates:

$$
\begin{gathered}
\xi^{5}(x, z)=\frac{z}{R} \zeta(x), \\
\xi_{\mu}(x, z)=\zeta_{\mu}(x)-\frac{1}{2} \frac{z^{2}}{R} \partial_{\mu} \zeta(x),
\end{gathered}
$$

and the transformation of the metric fluctuations is

$$
\delta h_{\mu \nu}=\frac{z^{2}}{R} \partial_{\mu} \partial_{\nu} \zeta+\frac{2}{R} \zeta \eta_{\mu \nu}-\partial_{\mu} \zeta_{\nu}-\partial_{\mu} \zeta_{\nu}
$$

Clearly, with an appropriate choice of $\zeta$ we can maintain the GGN gauge fixing conditions (10) and straighten one of the branes which will now be located at a constant $z$. This gauge choice for which the UV(IR) brane is straight will be called (GNIR)GNUV, generalized Gaussian normal gauge with respect to the UV(IR) brane. There is finally a third special generalized Gaussian normal gauge for which the 4D fluctuations are TT, i.e., traceless, $h=0$, and transverse, $\partial^{\sigma} h_{\sigma \mu}=0 .{ }^{3}$ This gauge will be denoted GNTT. Each gauge has its own advantage: in the GNTT gauge it will be easy to solve the bulk equations of motion while in the GNUV and GNIR gauges it will be easy to solve the boundary conditions. In the following sections we will explain how these different gauges are related to each other depending on the interactions and the matter localized on the branes. Finally note that the GNUV and GNIR gauges still possess usual 4D reparametrization invariance associated to the $\zeta^{\mu}(x)$.

\section{B. Boundary conditions in presence of brane mass term}

We now want to add some brane-localized interactions for the gravitational degrees of freedom. More precisely, we are interested in a localized mass term. Working in the generalized Gaussian system of coordinates in which the brane we want to add the mass term on is straight, the mass term is for simplicity chosen to be of the Fierz-Pauli form ${ }^{4}$ :

$$
\begin{aligned}
\mathcal{L}= & -\frac{1}{8} f_{\mathrm{UV}}^{4} \int d^{4} x\left(h_{\mu \nu} h^{\mu \nu}-h^{2}\right)_{\mid z=R}-\frac{1}{8} f_{I R}^{4} \frac{R^{4}}{R^{\prime 4}} \\
& \times \int d^{4} x\left(h_{\mu \nu} h^{\mu \nu}-h^{2}\right)_{\mid z=R^{\prime} .}
\end{aligned}
$$

Of course, since the mass terms (14) explicitly break general coordinate invariance, their forms will not be the same in a different system of coordinates and it will have to be determined by coordinate transformation from the appropriate GGN gauge. ${ }^{5}$ Note, in particular, that the two mass terms are not written in the same coordinate systems: the UV mass term is written in the GNUV gauge while the IR mass term is written in the GNIR gauge.

\footnotetext{
${ }^{3}$ To see this, first note that the residual gauge invariance can be used to set $h=0$ and $\partial^{\nu} h_{\nu \mu}=0$ at a point $z=z_{0}$. Then the ( $\mu 5)$ equation implies that $\partial_{\mu} h-\partial^{\nu} h_{\nu \mu}=0$ everywhere in the bulk. Using this result the (55) equation then implies $h^{\prime}=0$ everywhere. But since $h=0$ at a point, it is zero everywhere. Then $\partial^{\nu} h_{\mu \nu}=0$ in the bulk.

${ }^{4}$ The powers of the warp factor are determined by the requirement that in the coordinate system where $e^{A}=1$ at the IR brane say, the boundary condition on that brane is independent of the warp factor on the UV brane.

${ }^{5}$ In an abuse of language we will still refer to generalized coordinate transformations as "gauge transformations," even though this symmetry is explicitly broken.
} 
More general, non-Fierz-Pauli mass terms may also be considered. For brevity, the analysis of these more general mass terms is provided in Appendix B. The conclusions of this and subsequent sections is unchanged in the more general case: an additional state is present, but it decouples and the radion is always a ghost.

The effect of the mass term and additional matter localized on the brane is to modify the usual Neumann boundary condition for the metric fluctuations. The new boundary condition gets simplified on the GGN gauge where the brane is straight. In the GNUV gauge, the boundary condition at the UV brane is

$$
h_{\mu \nu}^{\prime}=-\kappa_{5}^{2}\left(S_{\mu \nu}^{\mathrm{UV}}-\frac{1}{3} \eta_{\mu \nu} S^{\mathrm{UV}}-f_{\mathrm{UV}}^{4} h_{\mu \nu}\right),
$$

where $S_{\mu \nu}^{\mathrm{UV}}$ is the stress-energy tensor for the matter localized on the UV brane, $S^{\mathrm{UV}}$ is its trace, $S_{\mu \nu}^{\mathrm{UV}} \eta^{\mu \nu}$. Similarly the boundary condition at the IR brane is

$$
h_{\mu \nu}^{\prime}=\kappa_{5}^{2}\left(S_{\mu \nu}^{\mathrm{IR}}-\frac{1}{3} \eta_{\mu \nu} S^{\mathrm{IR}}\right)-\kappa_{5}^{2} f_{\mathrm{IR}}^{4} \frac{R}{R^{\prime}} h_{\mu \nu}
$$

where warp factors have been absorbed into our definition of $S_{\mu \nu}^{\mathrm{IR}}$. These boundary conditions are obtained by varying the brane-localized mass term action given above, and then adding that to the left-hand side of the linearized Einstein equations. The boundary conditions are then obtained by requiring the cancellation of the boundary terms in the variation of the action (see for instance [19] for an analogous computation in gauge theories). For later convenience it is useful to introduce the following parameters that have the dimension of a mass

$$
\lambda_{\mathrm{IR}(\mathrm{UV})}=\kappa_{5}^{2} f_{\mathrm{IR}(\mathrm{UV})}^{4} .
$$

We can find the graviton Kaluza-Klein spectrum by solving the bulk equations for transverse and traceless excitations, supplemented by the above boundary conditions in the absence of matter on the branes. The presence of the brane mass terms lifts the zero mode from the spectrum. For instance, in the presence of a mass term on the IR brane only and in the limit $\lambda_{\mathrm{IR}} \ll R^{-1}$, the lightest spin-2 state has a mass given by (see Sec. IVA for details)

$$
m_{0}^{2} \sim 2 \frac{\kappa_{5}^{2} f_{\mathrm{IR}}^{4}}{R}\left(\frac{R}{R^{\prime}}\right)^{4} \sim \frac{2}{M_{P l}^{2}}\left(\frac{R}{R^{\prime}} f_{\mathrm{IR}}\right)^{4}
$$

where $M_{P l}^{2} \sim R / \kappa_{5}^{2}$ is approximately the four dimensional Planck mass.

The spectrum of light states with mass below the effective compactification scale $1 / R^{\prime}$ is seen to also contain a massless scalar, the radion. Part of this paper is devoted to the identification of the properties of this perturbation.

\section{State counting}

In this section we count the number of degrees of freedom in the gravity theory with a brane-localized Fierz-Pauli mass term. In the theory without such a mass term the spectrum consists of a massless spin-2 field with two polarizations, a radion and a tower of massive spin-2 Kaluza-Klein resonances with five polarizations each. Here we show that once the mass term is introduced the spectrum changes only in that the lightest spin-2 field is now massive and therefore has the five polarizations associated with a massive spin-2 particle.

In doing so we will make use of the important result that in the presence of the brane mass term and in the absence of any additional matter on the massive brane, the gauge GNIR(GNUV) is equivalent to the GNTT gauge. In subsequent sections we will also make use of this result.

Before demonstrating this, we note that this is similar to the situation with a massive $\mathrm{U}(1)$ vector boson $A_{\mu}$. There one has no gauge invariance, but a priori 4 degrees of freedom. However, the equations of motion for the vector boson imply that $\partial_{\mu} A^{\mu}=0$, eliminating one of the unphysical perturbations. Thus the theory describes three fluctuating degrees of freedom, the correct number for a massive spin-1 particle.

Similarly, a massive graviton in four dimensions $a$ priori describes 10 degrees of freedom, but has no gauge invariance. As with the massive vector boson, one finds that for the Fierz-Pauli mass term, the equations of motion imply that the metric is transverse and traceless. This eliminates five perturbations leaving five, which is the correct number for a massive spin-2 particle [12,22].

The situation with the brane-localized mass term is similar, but naively worse. This is because we are describing a five dimensional gravitational theory, which a priori has 15 degrees of freedom. Since the brane mass term explicitly breaks general coordinate invariance, there is a concern that additional states which were previously eliminated by the gauge invariance are now reintroduced. This would be a disaster for the model, just as for a massive graviton theory with a mass term in a non-Fierz-Pauli combination.

However, just as in the massive vector and graviton examples given above, we shall see that the equations of motion imply that for a Fierz-Pauli mass term defined in GNIR(GNUV) gauge, the metric is additionally TT in absence of any additional matter on the brane. Thus the only degrees of freedom are massive gravitons which involve only five physical polarizations and a massless radion associated with the movement of the brane. In addition, since this result will follow from the properties of the bulk equations of motion and the IR(UV) boundary condition, these conclusions are unchanged if a source is placed on the opposite UV(IR) brane.

To see this, let us consider the case of a mass term added on the IR brane and let us work in the GNIR gauge 
where the IR brane is straight. The IR boundary condition for the combination $H_{\mu} \equiv \partial^{\nu} h_{\nu \mu}-\partial_{\mu} h$ is

$$
\partial_{z} H_{\mu \mid z=R^{\prime}}=-\lambda_{\mathrm{IR}} \frac{R}{R^{\prime}} H_{\left.\mu\right|_{z}=R^{\prime}} .
$$

Now the $(\mu 5)$ equation implies that $\partial_{z} H_{\mu}=0$, so the lefthand side of the equation above vanishes identically. Since $\lambda_{\mathrm{IR}} \neq 0$, we learn that $H_{\mu}$ vanishes at the location of the IR brane. But since by the $(\mu 5)$ equation $H_{\mu}$ is constant in the bulk, we find that it actually vanishes identically. Using this result the (55) component of the bulk Einstein equations implies that $\partial_{z} h=0$ identically. Next consider the IR boundary condition again, but written as $\partial_{z} h_{\mu \nu \mid z=R^{\prime}}=-\lambda_{\mathrm{IR}} R / R^{\prime} h_{\mu \nu \mid z=R^{\prime}}$. The trace of this implies $\partial_{z} h_{\mid z=R^{\prime}}=-\lambda_{\mathrm{IR}} R / R^{\prime} h_{\mid z=R^{\prime}}$, which when combined with the previous result implies that $h_{\mid z=R^{\prime}}=0$. But $\partial_{z} h=0$, so $h$ vanishes in the bulk. From this result and $H_{\mu}=0$, it follows that $\partial^{\nu} h_{\nu \mu}=0$ identically.

The introduction of a brane-localized Fierz-Pauli mass term (14) therefore implies that the metric is transverse and traceless in the GGN gauge where the brane is straight (the GNIR gauge is also GNTT). Thus at the massive level there are only 5 degrees of freedom, corresponding to the helicity states of a massive graviton. The brane-localized Fierz-Pauli mass term does not introduce any additional massive degrees of freedom that were not already present in the RS model.

These results may be understood by noting that the model still has a large residual general invariance generated by coordinate transformations that vanish at the location of the brane. Referring to (9), this requirement implies $\xi_{\mid z=R^{\prime}}^{\mu}=0$ and $\xi_{\left.\right|_{z}=R^{\prime}}^{5}=0$. Thus the only gauge transformations explicitly broken by the brane mass term are those associated with the would-be zero mode graviton and the bending of the brane.

\section{TWO-POINT FUNCTION ANALYSIS}

In this section we obtain expressions for the graviton two-point correlator with external legs on the UV brane. ${ }^{6}$ We consider first the case with a mass term on the UV brane and then the case with a mass term on the IR brane. This calculation serves two purposes. We will be able to determine the extent to which observers on the UV brane find the theory to deviate from Einstein's gravity at any particular length scales. We will also be able to determine the masses of the light modes in the four dimensional effective theory. This is precisely the physics we are most interested in determining.

The metric perturbation in the linear approximation created by a source $S$ will be given by

\footnotetext{
${ }^{6}$ The analogous calculation for the case of a gauge field with a brane-localized mass term may be found in [23].
}

$$
h_{M N}(x, z)=\int d^{4} x^{\prime} d z^{\prime} \sqrt{|g|} \Delta_{M N}^{P Q}\left(X, X^{\prime}\right) S_{P Q}\left(x^{\prime}, z^{\prime}\right),
$$

where $\Delta$ is the Green's function. We are mostly interested in physics for an observer on the Planck brane and so we want to compute the two-point correlator with both external legs on the UV brane. Finding this correlator is equivalent to computing, in the GNUV coordinates, the metric perturbation on the UV brane as a response to a source localized on the UV brane too.

To obtain the two-point function we closely follow the work of Garriga and Tanaka [24]. The approach is to determine, at a point on the UV brane, the linearized gravitational field created by a source on the same UV brane. This can be related in a simple way to the graviton two-point correlator with external legs on the UV brane. The key observation is that it is convenient to first work in the GNTT gauge where the bulk equations are very simple. In this gauge the equations in the bulk reduce to

$$
\left(\square+\partial_{z}^{2}-\frac{3}{z} \partial_{z}\right) h_{\mu \nu}=0
$$

However, in this gauge both branes will in general not be straight and the bending of each brane provides an additional contribution to the stress tensors on the two boundaries, modifying the boundary conditions. The main effort of these sections is to determine this modification. With that information and the solution to the propagator in the GNTT gauge, we can readily evaluate the perturbation in the GNIR(GNUV) gauge .

We consider below two cases. In the first example, both the Fierz-Pauli mass term and the source are located on the UV brane, and the perturbation on the UV brane is determined. In the second, the Fierz-Pauli mass term is placed on the IR brane, with the source still placed on the UV brane.

\section{A. Fierz-Pauli mass term on the Planck brane}

In GN coordinates around the Planck brane (GNUV), the UV boundary condition is

$$
\partial_{z} h_{\mu \nu}^{\mathrm{GNUV}}=\lambda_{\mathrm{UV}} h_{\mu \nu}^{\mathrm{GNUV}}-\kappa_{5}^{2}\left(S_{\mu \nu}^{\mathrm{UV}}-\frac{1}{3} S^{\mathrm{UV}} \eta_{\mu \nu}\right) .
$$

with

$$
\lambda_{\mathrm{UV}}=\kappa_{5}^{2} f_{\mathrm{UV}}^{4}
$$

In order to be able to solve the Einstein's equations in the bulk, it is useful to perform a coordinate transformation in order to obtain a graviton perturbation that is transverse and traceless. The transformations (11) and (12) to TT coordinates yields a new boundary condition:

$$
\partial_{z} h_{\mu \nu}^{\mathrm{GNTT}}=\lambda_{\mathrm{UV}} h_{\mu \nu}^{\mathrm{GNTT}}-\kappa_{5}^{2} \Sigma_{\mu \nu}
$$

where the source term now includes a brane-bending contribution: 


$$
\begin{aligned}
\Sigma_{\mu \nu} & =S_{\mu \nu}-\frac{1}{3} \eta_{\mu \nu} S-\frac{2}{\kappa_{5}^{2}} \partial_{\mu} \partial_{\nu} \zeta \\
& +\frac{\lambda_{\mathrm{UV}}}{\kappa_{5}^{2}}\left(R \partial_{\mu} \partial_{\nu} \zeta+\frac{2}{R} \zeta \eta_{\mu \nu}-\partial_{\nu} \zeta_{\mu}-\partial_{\mu} \zeta_{\nu}\right) .
\end{aligned}
$$

The gauge parameters $\zeta$ and $\zeta^{\mu}$ are chosen so that in the new frame the metric is TT. Since $\Sigma_{\mu \nu}$ is the source for the metric perturbation in the GNTT gauge, it must be transverse and traceless too. This leads to the two conditions below on the gauge parameters:

(ii) $\quad-\frac{\kappa_{5}^{2}}{3} S-2 \square \zeta+\lambda_{\mathrm{UV}}\left(\frac{8}{R} \zeta+R \square \zeta-2 \partial_{\sigma} \zeta^{\sigma}\right)=0$.

\section{Massless case: $\lambda_{\mathrm{UV}}=0$}

To begin though, first suppose that no mass term is present. Then we should recover the results of Garriga and Tanaka [24]. In this case the TT conditions simplify and reduce to the single requirement that $\square \zeta=-\kappa_{5}^{2} S / 6$. In the GNTT coordinates, the source is thus related to the brane stress-energy tensor by:

$$
\Sigma_{\mu \nu}=S_{\mu \nu}-\frac{1}{3}\left(\eta_{\mu \nu}-\frac{\partial_{\mu} \partial_{\nu}}{\square}\right) S
$$

which is manifestly transverse and traceless. The solution for the metric fluctuations in the bulk is:

$$
h_{\mu \nu}^{\mathrm{GNTT}}(x, z)=-\kappa_{5}^{2} \int d^{4} x^{\prime} \Delta\left(x, x^{\prime} ; z, R\right) \Sigma_{\mu \nu}\left(x^{\prime}\right)
$$

where $\Delta$ is the Green's function for a scalar field in the Randall-Sundrum background. It satisfies the boundary conditions $\partial_{z} \Delta_{\left.\right|_{z=R}}=\partial_{z} \Delta_{I_{z=R^{\prime}}}=0$ and its solution may be found in the appendices. Back in the GN system the metric perturbation on the brane is

$$
h_{\mu \nu}^{\mathrm{GNUV}}(x, R)=h_{\mu \nu}^{\mathrm{GNTT}}(x, R)+\eta_{\mu \nu} \frac{\kappa_{5}^{2}}{3 R} \frac{1}{\square} S .
$$

where we have substituted for $\zeta$ and dropped terms involving longitudinal four dimensional derivatives. At long distances [see AppendixA, Eq. (A10)], $q \ll R^{-1}$, the propagator becomes

$$
\Delta\left(x, R ; x^{\prime}, R\right) \rightarrow \frac{2}{R} \frac{1}{1-\left(R / R^{\prime}\right)^{2}} \frac{1}{\square} \delta^{4}\left(x-x^{\prime}\right)
$$

and the metric perturbation in the GNUV coordinate is then [24] (again dropping terms involving longitudinal four dimensional derivatives)

$$
\begin{aligned}
h_{\mu \nu}^{\mathrm{GNUV}}= & -\frac{2 \kappa_{5}^{2}}{R} \frac{1}{1-\left(R / R^{\prime}\right)^{2}} \frac{1}{\square}\left(S_{\mu \nu}-\frac{1}{2} \eta_{\mu \nu} S\right) \\
& -\frac{\kappa_{5}^{2}}{3 R}\left(\frac{R}{R^{\prime}}\right)^{2} \frac{1}{1-\left(R / R^{\prime}\right)^{2}} \frac{\eta_{\mu \nu}}{\square} S .
\end{aligned}
$$

The crucial factor of $1 / 3$ from the gauge transformation $\zeta$ has been combined with the $1 / 3$ factor appearing in the trace part of the Green's function to obtain the correct factor of $1 / 2$ for a massless graviton [24]. The part that is left over is interpreted as due to the exchange of the radion, and appears here with the correct sign to describe a physical propagating particle.

The important point in this review of the results of Garriga and Tanaka is to draw attention to the technical reason for recovering the correct tensor structure of the massless graviton: the transformation between the TT and GN coordinate system involved a nonvanishing bending of the brane $\tilde{\xi}^{5}$. By contrast, a transformation involving $\zeta_{\mu}$ can only modify the part of the graviton propagator involving derivatives of the source, leaving the part involving the trace untouched. This is the situation encountered when, on the brane, a graviton mass term is turned on.

\section{Massive case: $\lambda_{\mathrm{UV}} \neq 0$}

For a nonzero mass term on the brane the first requirement (26) becomes nontrivial and implies that

$$
\square \zeta=0
$$

Decomposing the vector $\zeta_{\mu}$ into a scalar and a transverse part, $\zeta_{\mu}=\zeta_{\mu}^{T}-\partial_{\mu} \phi$ with $\partial^{\sigma} \zeta_{\sigma}^{T}=0$, the second condition (27) relates the scalar part to the brane stress-energy tensor:

$$
\square \phi=\frac{\kappa_{5}^{2}}{6 \lambda_{\mathrm{UV}}} S-\frac{4}{R} \zeta
$$

A consistent solution to the TT conditions is to set $\zeta=$ $\zeta_{\mu}^{T}=0$. This leads to the same expression (28) for the source $\Sigma_{\mu \nu}$ in terms of the boundary stress-tensor $S_{\mu \nu}$. Crucially though, the coordinate transformation needed to reach the GNTT frame now involves $\zeta_{\mu}$ rather than $\zeta$. Thus there is no brane bending to compensate the $5 \mathrm{D}$ structure of the brane propagator and we expect that gravity is never Einsteinian on the brane.

Going back to the GNUV frame using

$$
h_{\mu \nu}^{\mathrm{GNUV}}=h_{\mu \nu}^{\mathrm{GNTT}}-2 \partial_{\mu} \partial_{\nu} \phi
$$

we get a different expression for the metric fluctuation compared to when no brane mass term is present: 


$$
\begin{aligned}
h_{\mu \nu}^{\mathrm{GNUV}}(x, z)= & -\kappa_{5}^{2} \int d^{4} x^{\prime} \Delta\left(x, x^{\prime} ; z, R\right)\left[S_{\mu \nu}\right. \\
& \left.-\frac{1}{3}\left(\eta_{\mu \nu}-\frac{\partial_{\mu} \partial_{\nu}}{\square}\right) S\right]-\frac{\kappa_{5}^{2}}{3 \lambda_{\mathrm{UV}}} \frac{\partial_{\mu} \partial_{\nu}}{\square} S .
\end{aligned}
$$

Here $\Delta$ is the Green's function for a scalar field in the Randall-Sundrum background with a mass term on the UV brane. It satisfies the boundary conditions $\partial_{z} \Delta_{\left.\right|_{z=R}}=$ $\lambda_{\mathrm{UV}} \Delta_{\mathrm{I}_{z=R}}$ and $\partial_{z} \Delta_{\mathrm{I}_{z=R^{\prime}}}=0$. Its expression is given in the appendices.

As already mentioned, unlike the case with no brane mass term, here there is no brane bending. Thus the trace part of the propagator is the same in the GNTT and GNUV coordinate systems; in particular, the factor of $1 / 3$ does not change and Einstein gravity is not recovered.

In order to decouple the IR brane $\left(R / R^{\prime} \rightarrow 0\right)$ while keeping $f_{\mathrm{UV}}$ held fixed, we consider the long distance limit $q R \ll 1$ but keeping $q R^{\prime} \gg 1$ in order to probe the fifth dimension. Using the approximate expression (A12) of the propagator found in AppendixA for this limit, we arrive at

$$
\begin{aligned}
h_{\mu \nu}^{\mathrm{GNUV}}(x, R)= & -\frac{2 \kappa_{5}^{2}}{R} \frac{1}{\square-m^{2}} \\
& \times\left[S_{\mu \nu}-\frac{1}{3}\left(\eta_{\mu \nu}-\frac{\partial_{\mu} \partial_{\nu}}{m^{2}}\right) S\right],
\end{aligned}
$$

where $m^{2}=2 \lambda_{\mathrm{UV}} / R$. This is the correct propagator for a massive spin-2 particle [22], up to and including the derivative terms that scale as $m^{-2}$. At distances $R \ll r \ll$ $R^{\prime}$, it is not surprising then to find that the perturbation is dominated by the exchange of a single massive spin-2, with the exchange of the KK tower suppressed as in the Randall-Sundrum model.

At energy scales much below the compactification scale, $r \gg R^{\prime}$, the theory is four dimensional and the only light states are the radion and a massive graviton, for which there will be a van Dam-Veltman-Zakharov discontinuity. But in the limit just considered, where the IR brane is decoupled first, we cannot appeal to these arguments, as the theory is never four dimensional. Nevertheless, the result above, (37), demonstrates that even in this intrinsically five dimensional limit there is still a discontinuity.

Since in this limit there is a mass gap between the would-be zero mode graviton and the continuum of bulk gravitons that goes down to zero, following the reasoning of [25] one may suspect that the graviton studied here is unstable. In the appendices the two-point function is evaluated for complex, timelike $q^{2}$. There we find that the light graviton studied above does have a complex pole, with a lifetime $\Gamma$ given by $\Gamma / m \sim(m R)^{2}$. This lifetime is parametrically identical to the scalar example studied in [25]. Since here though $R^{-1} \geqslant 10^{-3} \mathrm{eV}$ and $m \lesssim H_{0}$, the lifetime is much longer than the age of the universe.

Finally, one may be puzzled by the absence of any term in (36) that could possibly be interpreted as due to the exchange of a radion with nonderivative couplings. As shown at the end of Sec. IV, the radion is normalizable and physical (not a ghost), and has a wave function that is localized about the IR brane. However, in contrast to RS, here the radion wave function vanishes at the UV brane and has only derivative couplings to sources located there. Therefore it does not contribute to the two-point correlation function of two conserved sources located at the UV brane.

\section{B. Fierz-Pauli mass term on the infrared brane}

We consider the case where the Fierz-Pauli mass term is on the IR brane. The source remains on the UV brane.

The first observation is that in the GGN coordinate system with respect to the IR brane (GNIR), the metric is additionally TT, since there is no source on the IR brane (see Sec. II). Thus the metric satisfies (21) in the bulk, with the IR boundary condition

$$
\partial_{z} h_{\mu \nu}^{\mathrm{GNTT}}=-\lambda_{\mathrm{IR}} \frac{R}{R^{\prime}} h_{\mu \nu}^{\mathrm{GNTT}},
$$

with

$$
\lambda_{\mathrm{IR}}=\kappa_{5}^{2} f_{\mathrm{IR}}^{4} .
$$

In this gauge however the UV brane is bent, due to the source located there. To determine the UV boundary condition in the GNTT gauge, we first consider the GN coordinates with respect to the UV brane (GNUV) and then perform a coordinate transformation. In the GNUV gauge, the UV boundary condition is

$$
\partial_{z} h_{\mu \nu}^{\mathrm{GNUV}}=-\kappa_{5}^{2}\left(S_{\mu \nu}-\frac{1}{3} \eta_{\mu \nu} S\right) .
$$

Inserting the transformations (11) and (12) relating the GNUV gauge and GNTT gauge metric into the above boundary condition gives the desired UV boundary condition in the GNTT gauge:

$$
\partial_{z} h_{\mu \nu}^{\mathrm{GNTT}}=-\kappa_{5}^{2} \Sigma_{\mu \nu},
$$

where the source term now includes a brane-bending contribution:

$$
\Sigma_{\mu \nu}=S_{\mu \nu}-\frac{1}{3} \eta_{\mu \nu} S-2 \kappa_{5}^{-2} \partial_{\mu} \partial_{\nu} \zeta .
$$

As before, requiring that this source is transverse and traceless fixes $\square \zeta=-\kappa_{5}^{2} S / 6$. In GNTT gauge then, the UV source is

$$
\Sigma_{\mu \nu}=S_{\mu \nu}-\frac{1}{3}\left(\eta_{\mu \nu}-\frac{\partial_{\mu} \partial_{\nu}}{\square}\right) S .
$$

The solution for the metric perturbation in the bulk is 


$$
h_{\mu \nu}^{\mathrm{GNTT}}(x, z)=-\kappa_{5}^{2} \int d^{4} x^{\prime} \Delta\left(x, x^{\prime} ; z, R\right) \Sigma_{\mu \nu}\left(x^{\prime}\right),
$$

where $\Delta$ satisfies the Green's function equation

$$
\left(\square+\partial_{z}^{2}-\frac{3}{z} \partial_{z}\right) \Delta\left(x, z ; x^{\prime}, z^{\prime}\right)=\frac{z^{3}}{R^{3}} \delta^{(4)}\left(x-x^{\prime}\right) \delta\left(z-z^{\prime}\right),
$$

with boundary conditions $\partial_{z} \Delta_{\mid z=R}=0$ and $\partial_{z} \Delta_{\mid z=R^{\prime}}+$ $\lambda_{\mathrm{IR}}\left(R / R^{\prime}\right) \Delta_{\mid z=R^{\prime}}=0$. The Green's function $\Delta$ is given in the appendices, and more details can be found there.

Back in the GNUV coordinate system, the metric perturbation is

$$
h_{\mu \nu}^{\mathrm{GNUV}}(x, R)=h_{\mu \nu}^{\mathrm{GNTT}}(x, R)+\eta_{\mu \nu} \frac{\kappa_{5}^{2}}{3 R} \frac{1}{\square} S,
$$

where we have substituted for $\zeta$ and dropped terms involving four dimensional derivatives. Using results (A7)(A9) obtained in AppendixA, at long distances where we cannot probe the KK excitations, $q R^{\prime} \ll 1$, the asymptotic form of the propagator is

$$
\Delta\left(x, R ; x^{\prime}, R\right) \rightarrow \frac{\mathcal{N}}{\square-m^{2}} \delta^{(4)}\left(x-x^{\prime}\right),
$$

where $\mathcal{N}$ and $m^{2}$ may be found in the appendices. Focusing on nonderivative terms, in this limit the GNUV metric perturbation reduces to

$$
\begin{aligned}
h_{\mu \nu}^{\mathrm{GNUV}}(x, R)= & -\kappa_{5}^{2} \frac{\mathcal{N}}{\square-m^{2}}\left(S_{\mu \nu}-\frac{1}{3} \eta_{\mu \nu} S\right) \\
& +\eta_{\mu \nu} \frac{\kappa_{5}^{2}}{3 R} \frac{1}{\square} S .
\end{aligned}
$$

The last term is due to the gauge transformation between the GNTT and GNUV coordinates and is independent of the IR boundary mass term.

In this limit the first two terms describe the exchange of a massive graviton, and the last term describes the exchange of a massless scalar (the radion). But the sign of the last term implies that the radion is a ghost. This conclusion is independent of the size of the IR FierzPauli mass, $f_{\mathrm{IR}}$.

An interesting limit to look at is when 4D momenta can only probe the lightest graviton and not the regular KK excitations: $\quad m \ll q \ll 1 / R^{\prime}, \quad$ then $\quad \mathcal{N} \rightarrow 2 /[1-$ $\left.\left(R / R^{\prime}\right)^{2}\right] / R$, and the metric perturbation on the UV brane becomes (again dropping terms involving longitudinal $4 \mathrm{D}$ derivatives)

$$
\begin{aligned}
h_{\mu \nu}^{\mathrm{GNUV}}= & -2 \frac{\kappa_{5}^{2}}{R} \frac{1}{1-\left(R / R^{\prime}\right)^{2}} \frac{1}{\square}\left(S_{\mu \nu}-\frac{1}{2} \eta_{\mu \nu} S\right) \\
& -\frac{\kappa_{5}^{2}}{3 R} \frac{\eta_{\mu \nu}}{1-\left(R / R^{\prime}\right)^{2}} \frac{R^{2}}{R^{\prime 2}} \frac{1}{\square} S,
\end{aligned}
$$

with $\mathcal{O}\left(\lambda_{\mathrm{IR}} R\right)$ corrections not included. This expression goes smoothly over to the result for Randall-Sundrum.
Finally, we can see that for UV brane observers Einstein gravity is recovered at distances shorter than the compactification scale. Consider $q R^{\prime} \gg 1$ but $q R \ll$ 1. In this limit all dependence on the IR brane disappears. Using results (A11) obtained in AppendixA, the perturbation on the UV brane is indeed found to be (still dropping terms involving longitudinal 4D derivatives)

$$
h_{\mu \nu}^{\mathrm{GNUV}}(x, R)=-\frac{2 \kappa_{5}^{2}}{R} \frac{1}{\square}\left(S_{\mu \nu}-\frac{1}{2} \eta_{\mu \nu} S\right) .
$$

Nevertheless when $f_{\mathrm{IR}} \neq 0$, the theory has a ghost which is responsible for the recovery of $4 \mathrm{D}$ gravity on the Planck brane. We will show in Sec. IV that the ghost mode is the radion.

\section{MODE DECOMPOSITION ANALYSIS}

\section{A. Spin-2 excitations: graviton mass spectrum}

We are first interested in the spectrum and the KK decomposition of the spin-2 excitations. In the GNTT gauge, the bulk equations of motion do not couple the different polarizations and thus simply reduce to the scalar equation of the form:

$$
\square \phi+\phi^{\prime \prime}-\frac{3}{z} \phi^{\prime}=0 .
$$

The mode decomposition can be written as

$$
\phi(x, z)=\sum_{n}\left(\frac{z}{R}\right)^{2} \psi_{n}(z) \phi_{n}(x),
$$

the wave functions $\psi_{n}(z)$ then satisfy a Bessel equation of order $\nu=2\left(m_{n}\right.$ is the 4D mass of the eigenmode):

$$
\psi_{n}^{\prime \prime}+\frac{1}{z} \psi_{n}^{\prime}+\left(m_{n}^{2}-\frac{4}{z^{2}}\right) \psi_{n}=0,
$$

whose solutions are

$$
\psi_{m}(z)=A_{n} J_{2}\left(m_{n} z\right)+B_{n} Y_{2}\left(m_{n} z\right),
$$

where the two constants are fixed by the boundary and the normalization conditions. The boundary conditions for the spin- 2 excitations are unaffected by coordinate transformations of the form (11) and (12) and therefore take the same form within the GNTT gauge as in the GNUV and GNTT gauges:

$$
\begin{gathered}
{\left[\psi_{n}^{\prime}+\left(\frac{2}{R}-\lambda_{\mathrm{UV}}\right) \psi_{n}\right]_{\mid z=R}=0} \\
{\left[\psi_{n}^{\prime}+\left(\frac{2}{R}+\lambda_{\mathrm{IR}}\right) \frac{R}{R^{\prime}} \psi_{n}\right]_{I_{z=R^{\prime}}}=0 .}
\end{gathered}
$$

Clearly as soon as a nonvanishing mass term is turned on at either brane, the would-be massless mode, $\psi=R^{2} / z^{2}$, cannot satisfy the boundary conditions: the massless mode gets lifted by the brane-localized masses. For the massive modes, the boundary conditions (55) and (56) 
lead to the quantization equation:

$$
\begin{aligned}
& \frac{m_{n} J_{1}\left(m_{n} R\right)-\lambda_{\mathrm{UV}} J_{2}\left(m_{n} R\right)}{m_{n} Y_{1}\left(m_{n} R\right)-\lambda_{\mathrm{UV}} Y_{2}\left(m_{n} R\right)} \\
& \quad=\frac{m_{n} J_{1}\left(m_{n} R^{\prime}\right)+\lambda_{\mathrm{IR}} \frac{R}{R^{\prime}} J_{2}\left(m_{n} R^{\prime}\right)}{m_{n} Y_{1}\left(m_{n} R^{\prime}\right)+\lambda_{\mathrm{IR}} \frac{R}{R^{\prime}} Y_{2}\left(m_{n} R^{\prime}\right)} .
\end{aligned}
$$

Let us examine the solutions of this quantization equation in the two special cases when a single brane mass term is turned on at either the IR or the UV brane.

\section{Mass term on the IR brane $\left(\lambda_{\mathrm{UV}}=0, \lambda_{\mathrm{IR}} \neq 0\right)$}

Assuming that $m_{n} R^{\prime} \ll 1$ and expanding the Bessel functions near the origin, we find that the lightest mode has a mass approximately given by

$$
m_{0}^{2}=\frac{8}{R^{\prime 2}} \frac{\lambda_{\mathrm{IR}} R}{4+\lambda_{\mathrm{IR}} R}\left(\frac{R}{R^{\prime}}\right)^{2} .
$$

There is a gap of order $R / R^{\prime}$ between this lowest mode and the regular KK modes that have mass

$$
m_{n} \sim \frac{x_{n}}{R^{\prime}}
$$

where $x_{n} \sim(n+1 / 4) \pi$ are the roots of the $J_{1}$ Bessel function: $J_{1}\left(x_{n}\right)=0$.

The normalization of the wave function can be found analytically using the Wronskian method (see for instance Ref. [26]). We found

$$
\psi_{n}(z) \equiv \psi_{m_{n}}=\mathcal{N}_{n}\left[J_{2}\left(m_{n} z\right)-\frac{J_{1}\left(m_{n} R\right)}{Y_{1}\left(m_{n} R\right)} Y_{2}\left(m_{n} z\right)\right]
$$

with

$$
\begin{aligned}
\frac{1}{\mathcal{N}_{n}^{2}}= & \frac{2}{\pi^{2} m_{n}^{2} R}\left(\frac{\lambda_{\mathrm{IR}}^{2} R^{2}+4 \lambda_{\mathrm{IR}} R+m_{n}^{2} R^{\prime 2}}{\left[\lambda_{\mathrm{IR}} R Y_{2}\left(m R^{\prime}\right)+m_{n} R^{\prime} Y_{1}\left(m R^{\prime}\right)\right]^{2}}\right. \\
& \left.-\frac{1}{Y_{1}^{2}\left(m_{n} R\right)}\right) .
\end{aligned}
$$

\section{Mass on the $\boldsymbol{U V}$ brane $\left(\lambda_{\mathrm{IR}}=0, \lambda_{\mathrm{UV}} \neq 0\right)$}

For a large warp factor, $R^{\prime} / R \gg 1$, the quantization Eq. (57) can be approximately simplified to

$$
\left[m_{n} Y_{1}\left(m_{n} R\right)-\lambda_{\mathrm{UV}} Y_{2}\left(m_{n} R\right)\right] J_{1}\left(m_{n} R^{\prime}\right)=0,
$$

the solutions of which form the regular KK modes again obtained from the roots, $x_{n}$, of the $J_{1}$ Bessel function:

$$
m_{n} \sim \frac{x_{n}}{R^{\prime}}
$$

On top of this tower, there is another mode that is continuously connected to the massless graviton when $\lambda_{\mathrm{UV}}$ goes to zero. For this special mode to be parametrically lighter than the regular KK modes, the mass term added on the UV brane must be small enough. More precisely, when $\lambda_{\mathrm{UV}} R \ll\left(R / R^{\prime}\right)^{2}$, then the mass of the lightest graviton is approximated by

$$
m_{0}^{2}=\lambda_{\mathrm{UV}} R\left(\frac{R^{\prime}}{R}\right)^{2} \frac{2}{R^{\prime 2}}
$$

The mass of this mode can become larger than the compactification scale when $1 / R^{\prime}$ gets smaller and smaller and $\lambda_{\mathrm{UV}} R$ held fixed. In the limit $1 / R^{\prime} \rightarrow 0$, it becomes non-normalizable and is no longer in the spectrum. Instead, a resonance with a finite lifetime is found (see Appendix 3).

\section{B. The radion as a ghost}

\section{The radion wave function}

To provide further evidence that the interpretation of the two-point function obtained previously is indeed correct, in this section the radion's wave function is determined and its effective action computed. The principal result of this section is a confirmation that when the FierzPauli mass term is on the IR brane the radion is a ghost.

This conclusion is unchanged even if we allow for a non-Fierz-Pauli mass term on the IR brane. The details of that analysis are provided in Appendix B.

In fact, the wave function is rather straightforward to obtain in the GNTT coordinate system. By Lorentz covariance the metric describing massless scalar fluctuations $\Phi^{i}$ must be proportional to $\partial_{\mu} \partial_{\nu} \Phi^{i}$ with $\square \Phi^{i}=0$. Inspecting Einstein's equations in GNTT coordinates the general solution is trivial to obtain. It is :

$$
h_{\mu \nu}^{\mathrm{GNTT}}=-\frac{z^{4}}{2 R^{3}} \partial_{\mu} \partial_{\nu} f+\partial_{\mu} \partial_{\nu} \phi
$$

where $f$ and $\phi$ are massless scalars. At this point the boundary conditions are not yet imposed, since the branes are in general bent. For future reference, in this system the $\mathrm{UV}$ and IR branes are located at $z_{\mathrm{UV}}=R-\zeta(x)$ and $z_{\mathrm{IR}}=R^{\prime}-\left(R^{\prime} / R\right) \phi_{2}(x)$ (the normalization is chosen for later convenience).

As a check on this result, the wave function of Charmousis, Gregory and Rubakov (CGR) [27] for the radion is now recovered. To do this, transform to the GNUV coordinate system $z^{(\mathrm{GNUV})}=z+(z / R) \zeta$ where the UV brane is straight and located at $z_{\mathrm{UV}}=R$. The IR brane is located at $z_{\mathrm{IR}}=R^{\prime}-\left(R^{\prime} / R\right) \eta(x)$ with $\eta=$ $\phi_{2}-\zeta$. The new metric is

$$
h_{\mu \nu}^{\mathrm{GNUV}}=-\frac{z^{4}}{2 R^{3}} \partial_{\mu} \partial_{\nu} f+\partial_{\mu} \partial_{\nu} \phi+\frac{z^{2}}{R} \partial_{\mu} \partial_{\nu} \zeta+\frac{2}{R} \eta_{\mu \nu} \zeta .
$$

The UV boundary condition in this system is $\partial_{z} h_{\mu \nu}^{\mathrm{GNUV}}=$ 0 and determines the unknown function $\zeta$ to be $\zeta=f$ with $\phi$ still unconstrained. This gives the CGR solution 


$$
h_{\mu \nu}^{\mathrm{GNUV}}=\left(-\frac{z^{4}}{2 R^{3}}+\frac{z^{2}}{R}\right) \partial_{\mu} \partial_{\nu} f+\frac{2}{R} \eta_{\mu \nu} f+\partial_{\mu} \partial_{\nu} \phi .
$$

Actually this is not quite the CGR solution, for here there is the additional term proportional to $\phi$. In the RS1 model with no brane mass term, this term can be gauged away. This is because in the restricted GN coordinate system with the Planck brane straight and no mass term on any brane, there is a residual gauge invariance given by $\zeta_{\mu}=$ $\partial_{\mu} \phi / 2$ that may be used.

When the IR brane mass term is present this gauge invariance does not exist and $\phi$ cannot be eliminated in this way. It is seen below that in unitary gauge this mode is eliminated by the IR boundary condition. In a nonunitary gauge $\phi$ corresponds to the Goldstone boson associated to the longitudinal component of the graviton.

To determine the radion function in the GNIR coordinate system with the IR brane straight, it is useful to recall the following result, derived previously in Sec. II. Namely, when the IR brane mass term is present the metric in these coordinates is in addition TT. But the most general solution of Einstein's equations for a massless scalar in GNTT gauge was already obtained. It is

$$
h_{\mu \nu}^{\mathrm{GNIR}}=h_{\mu \nu}^{\mathrm{GNTT}}=\left(-\frac{z^{4}}{2 R^{3}}+\gamma\right) \partial_{\mu} \partial_{\nu} f+\partial_{\mu} \partial_{\nu} \phi .
$$

(Compared with previous notation here we have defined $\phi$ slightly differently and pulled out a factor $\gamma$ ). This contains two massless scalars. One of these is the radion and the other, as mentioned above, is the Goldstone boson corresponding to the longitudinal component of the graviton. In the unitary gauge the IR boundary condition is $\partial_{z} h_{\mu \nu}^{\mathrm{GNTT}}=-\lambda_{\mathrm{IR}}\left(R^{\prime} / R\right) h_{\mu \nu}^{\mathrm{GNTT}}$. This determines $\phi$ in terms of $f$, or equivalently, determines $\gamma$ after setting $\phi=0$.

In a nonunitary gauge $\phi$ is no longer zero. But the IR boundary condition is then modified due to an extra term coming from the Goldstone boson, which may be identified with $\phi$.

In summary, in GNIR gauge (68), with $\phi=0$, is the radion wave function.

\section{The radion kinetic term}

The wave function obtained above is not very useful for determining the effective action, since the UV brane is not straight. We would like to compute the effective action in a coordinate system with both branes straight.

To do this, begin in the GNIR gauge. The radion wave function is given by (68), and the UV brane is located at $z=R-\zeta(x)$. We first need to find the position of the UV brane in the GNIR gauge. But this has been determined already, since here the UV boundary condition is the same as in RS1. Hence Eq. (66) and (67) yield $\zeta=f$.

Next we straighten both branes. To do this, it is easiest to start again in the GNIR $=$ GNTT coordinates where the IR brane is straight and the radion wave function is given by (68), and perform a final coordinate transformation of the form

$$
z^{(\mathrm{rad})}=z-\frac{z F(z)}{R} f(x)
$$

maintaining $h_{\mu 5}=0$ but not $h_{55}=0$. $F$ is an arbitrary function with the only restriction that both branes are now straight, which implies $F\left(R^{\prime}\right)=0$ and $F(R)=-1$. The normalization of the radion wave function will be found to depend only on the values of $F$ at the location of the branes, and not on its particular shape. The metric in this final coordinate system is

$$
h_{\mu \nu}^{(\mathrm{rad})}=c(z) \partial_{\mu} \partial_{\nu} f-\frac{2}{R} F(z) f \eta_{\mu \nu}, \quad h_{55}^{(\mathrm{rad})}=\frac{2 z}{R} F^{\prime} f,
$$

with

$$
c(z)=-\frac{z^{4}}{2 R^{3}}+\gamma-2 \int^{z} d z^{\prime} \frac{z^{\prime}}{R} F\left(z^{\prime}\right) .
$$

It is straightforward to verify that for arbitrary $F$, restricted to the boundary conditions $F\left(R^{\prime}\right)=0$ and $F(R)=-1$, this expression satisfies the equations in the bulk and also both boundary conditions.

An inspection of this solution indicates a significant difference between the radion here and in the RS1 model. Here the brane mass term forces the non-TT part of the radion wave function to vanish at the IR brane. That is, here $F\left(R^{\prime}\right)=0$. This is equivalent to the requirement that the metric be traceless in the GNIR coordinate system. This fact is instrumental in turning the radion into a ghost.

To determine the radion kinetic term we want to integrate out the short-distance variation of the metric. To do this we follow the methodology of $[6,28]$. For full details, such as carefully adding the Gibbons-Hawking boundary term and seeing that the massive gravitons decouple, or for the more general case of a non-Fierz-Pauli mass term, see Appendix B. Here we quote the main results for the case of the Fierz-Pauli mass term. Expanding the action to quadratic order gives

$$
\begin{aligned}
S_{\text {eff }}= & -\frac{1}{4 \kappa_{5}^{2}} \int d^{4} x d z\left(\frac{R}{z}\right)^{3} h^{A B} E_{A B}\left[h_{C D}\right] \\
& + \text { boundary terms }+ \text { brane mass term. }
\end{aligned}
$$

Next we insert the expression (70) for the radion into the action, without using its four dimensional equations of motion. Since nonderivative terms in the wave function satisfy Einstein's equation, we are guaranteed that the integrand is of the form $f \square f$. It is then a matter of collecting terms appearing in the linearized Einstein equations which have four dimensional derivatives. Then we find ${ }^{7}$

\footnotetext{
${ }^{7}$ We have added in the boundary terms. For more details see Appendix B.
} 


$$
\begin{aligned}
S_{\text {eff }}= & -\frac{1}{4 \kappa_{5}^{2}} \int d^{4} x \int_{R}^{R^{\prime}} d z\left(\frac{R}{z}\right)^{3} h^{A B} E_{A B}\left[h_{C D}\right] \\
& + \text { brane mass term }-\frac{1}{8 \kappa_{5}^{2}} \int d^{4} x\left(\frac{R}{z}\right)^{3}\left[h^{\mu \nu} h_{\mu \nu}^{\prime}\right. \\
& \left.-h h^{\prime}\right]\left.\right|_{R} ^{R^{\prime}}+\left.\frac{3}{8 \kappa_{5}^{2}} \int d^{4} x \frac{R^{3}}{z^{4}} h_{55} h\right|_{R} ^{R^{\prime}} \\
= & -\frac{3}{2 \kappa_{5}^{2} R} \int d^{4} x f \square f \int_{R}^{R^{\prime}} d z F^{\prime} \\
= & -\frac{3}{2 \kappa_{5}^{2} R}\left[F\left(R^{\prime}\right)-F(R)\right] \int d^{4} x f \square f
\end{aligned}
$$

Since $F\left(R^{\prime}\right)=0$ and $F(R)=-1$, the radion kinetic term is

$$
S_{\text {eff }}=-\frac{3}{2 \kappa_{5}^{2} R} \int d^{4} x f \square f
$$

which has the wrong sign. The radion is a ghost!

Repeating this calculation for RS1 provides an independent check on the overall sign, since here the radion is known to be healthy. In fact, the formula is the same and the boundary condition in the UV is the same, but the IR boundary condition is different. So $F_{\mathrm{RS} 1}(R)=-1$ and $F_{\mathrm{RS} 1}\left(R^{\prime}\right)=-\left(R^{\prime 2} / R^{2}\right)$, implying that the radion has a physical kinetic term.

It is straightforward to repeat this exercise when the Fierz-Pauli mass term is on the UV brane. The radion wave function in the GNUV coordinates is still given by (68), but here the integration constant $\gamma$ is different in order to satisfy the UV boundary condition. Just as in the previous example, here it is the IR boundary condition that determines the position of the IR brane in the GNUV gauge. One finds $\zeta=\left(R^{\prime 2} / R^{2}\right) f$, which not surprisingly, is the same as in RS1. Then starting from GNUV coordinates, we straighten the IR brane, keeping the UV brane straight and maintaining $h_{\mu 5}=0$. In the notation of (69), this requires $F(R)=0$ and $F\left(R^{\prime}\right)=-\left(R^{\prime 2} / R^{2}\right)$. The computation of the radion kinetic term proceeds as before, and one arrives at (73). Here though one finds that the radion has a healthy kinetic term and is not a ghost. The radion wave function is also peaked at the IR brane and in the limit that the IR brane is decoupled the radion is not normalizable. All of these properties of the radion are also found to occur in the RS model. These results with a UV mass term are not surprising, since all we are doing here is adding a small perturbation on the UV brane where in the RS model the radion already had an exponentially small support.

Finally, a minor puzzle raised in Sec. III is now resolved. In the computation (36) of the perturbation due to a source on the UV brane there was no term that could be interpreted as due to the exchange of a radion with nonderivative couplings. The reason for this is that the nonderivative component of the radion appearing in $h_{\mu \nu}-$ see (70) - vanishes on the UV brane since in this model $F(R)=0$.

These conclusions generalize to the case with a non-Fierz-Pauli mass term. Here we summarize the results of Appendix B. If the mass term is on the IR brane, the radion is still a ghost but now there is an additional state that is decoupled and has a physical kinetic term. If the mass is on the UV brane, the radion still has a physical kinetic term, but now there is an additional state that is a ghost. Both of these results are not surprising from the perspective of the AdS/CFT correspondence.

\section{CONCLUSIONS}

We have investigated the physics of brane-localized mass terms for the graviton in warped backgrounds. We have performed a linearized analysis of the graviton twopoint correlator as well as a mode decomposition of the five dimensional theory. We find that if the mass term is localized on the UV brane, observers on that brane see physics similar to that of a massive graviton in four dimensions. One important distinction, however, is that if the graviton mass is larger than the mass of the lightest Kaluza-Klein modes it can now decay off the brane into these states.

A Fierz-Pauli mass term for the graviton on the IR brane reproduces Einstein's gravity for observers localized on the UV brane at length scales shorter than the inverse mass of the lightest Kaluza-Klein modes. At length scales longer than this the spectrum consists of a massive graviton and a ghost. It is the radion field which is the ghost.

For a non-Fierz-Pauli mass term on the IR brane there is an additional, physical state in the theory. But the radion field is still a ghost. For a non-Fierz-Pauli mass term on the UV brane the radion is physical but now there is an additional state in the theory that is a ghost.

It is of interest to consider whether there are simple modifications of this theory that could evade this problem. In models of latticized gravity [29] the radion excitation is absent, but unitarity is still maintained up to scales larger than the compactification scale. It is therefore conceivable that a latticized version of the model we have considered could be a successful realization of a theory which modifies gravity at long distances.

\section{ACKNOWLEDGMENTS}

We would like to thank Markus Luty, Jihad Mourad, and Mark Wise for useful discussions. Z.C. and M. G. would like to thank the hospitality of Saclay. M. G. and C. G. would like to thank the hospitality of Lawrence Berkeley National Laboratory. C. G. and L. P. thank the Aspen Center for Physics for its hospitality while part of this work was completed. The work of M. G. is supported by the U.S. Department of Energy under Contract 
No. DE-FG03-92-ER40701. C. G. and L. P. are supported in part by the RTN European Program HPRN-CT-200000148 and the ACI Jeunes Chercheurs 2068.

\section{APPENDIX A: SCALAR PROPAGATOR}

\section{General expression}

The scalar Green's function equation with mass terms localized on the UV and IR branes and a source at $z=z^{\prime}$ is solution of the bulk equation

$$
\left(\partial_{z}^{2}-\frac{3}{z} \partial_{z}-q^{2}\right) \Delta=\frac{z^{3}}{R^{3}} \delta\left(z-z^{\prime}\right),
$$

supplemented by the two boundary conditions at the UV and IR branes:

$$
\partial_{z} \Delta_{\mid R}=\lambda_{\mathrm{UV}} \Delta_{\mid R} \quad \text { and } \quad \partial_{z} \Delta_{\mid R^{\prime}}=-\lambda_{\mathrm{IR}} \frac{R}{R^{\prime}} \Delta_{\mid R^{\prime}} .
$$

The Green's function solution to this differential equation is obtained by first solving the homogeneous equation to the left $\left(z<z^{\prime}\right)$ and to the right $\left(z>z^{\prime}\right)$ of the source. This gives two solutions $\Delta_{<}$and $\Delta_{>}$, respectively, each having two undetermined integration constants. The boundary conditions at the UV and IR branes fixes the ratio of the integration constants in each region. Matching these two solutions at $z=z^{\prime}$ requires continuity of the solution,

$$
\Delta_{<\mid z=z^{\prime}}=\Delta_{>\mid z=z^{\prime}}
$$

and the source equation implies

$$
\partial_{z}\left(\Delta_{>}-\Delta_{<}\right)_{\mid z=z^{\prime}}=\frac{z^{13}}{R^{3}} .
$$

The first condition determines the ratio of integration constants between the left and right regions, and the second condition fixes their overall normalization. The unique solution, for spacelike $q^{2}$, is

$$
\begin{aligned}
\Delta\left(z, z^{\prime}\right)= & \frac{\left(z z^{\prime}\right)^{2}}{R^{3}} \frac{1}{\alpha \delta-\beta \gamma}\left[\alpha K_{2}\left(q z_{>}\right)-\beta I_{2}\left(q z_{>}\right)\right] \\
& \times\left[\gamma K_{2}\left(q z_{<}\right)-\delta I_{2}\left(q z_{<}\right)\right],
\end{aligned}
$$

where $z_{<}=\operatorname{Min}\left(z, z^{\prime}\right)$ and $z_{>}=\operatorname{Max}\left(z, z^{\prime}\right)$ and with

$$
\begin{aligned}
& \alpha=I_{1}\left(q R^{\prime}\right)+\frac{\lambda_{\mathrm{IR}} R}{q R^{\prime}} I_{2}\left(q R^{\prime}\right), \\
& \beta=-K_{1}\left(q R^{\prime}\right)+\frac{\lambda_{\mathrm{IR}} R}{q R^{\prime}} K_{2}\left(q R^{\prime}\right), \\
& \gamma=-I_{1}(q R)+\frac{\lambda_{\mathrm{UV}}}{q} I_{2}(q R), \\
& \delta=K_{1}(q R)+\frac{\lambda_{\mathrm{UV}}}{q} K_{2}(q R) .
\end{aligned}
$$

\section{Mass on the IR brane $\left(\lambda_{\mathrm{UV}}=0, \lambda_{\mathrm{IR}} \neq 0\right)$}

In the long distance limit $q R^{\prime} \ll 1$, by expanding the Bessel functions around the origin we get the leading form of the propagator with both legs on the UV brane

$$
\Delta\left(R, R, q^{2}\right) \rightarrow \frac{\mathcal{N}}{\square-m^{2}} \delta^{(4)}\left(x^{\prime}-x\right),
$$

with

$$
\mathcal{N}=\frac{2}{R} \frac{1+\frac{1}{4}\left(1-\frac{R^{4}}{R^{\prime 4}}\right) \lambda_{\mathrm{IR}} R}{1-\frac{R^{2}}{R^{\prime 2}}+\frac{1}{4}\left(1-2 \frac{R^{2}}{R^{2}}\right) \lambda_{\mathrm{IR}} R},
$$

and

$$
m^{2}=\frac{8 \lambda_{\mathrm{IR}}}{R\left(4+\lambda_{\mathrm{IR}} R\right)}\left(\frac{R}{R^{\prime}}\right)^{4} .
$$

to leading order in $R / R^{\prime}$. We recover the expression (58) for the lightest graviton found in Sec. IVA. As another check, note that in the limit $\lambda_{\mathrm{IR}} R \rightarrow 0$, the RS1 result $\mathcal{N} \rightarrow 2 /\left[1-\left(R / R^{\prime}\right)^{2}\right] / R$, is recovered:

$$
\Delta\left(R, R, q^{2}\right) \rightarrow \frac{2}{R\left(1-\frac{R^{2}}{R^{2}}\right)} \frac{1}{\square} \delta^{(4)}\left(x^{\prime}-x\right),
$$

In the limit $\lambda_{\mathrm{IR}} R \ll 1$, we obtain $m^{2}=$ $2 \lambda_{\mathrm{IR}}\left(R / R^{\prime}\right)^{4} / R$. Using $\lambda_{\mathrm{IR}}=\kappa_{5}^{2} f_{\mathrm{IR}}^{4}$, where $f_{\mathrm{IR}}^{4}$ is the coefficient of the Fierz-Pauli (bare) mass term, gives $m^{2}=$ $2\left(f_{\mathrm{IR}} R / R^{\prime}\right)^{4} / M_{P l}^{2}$, the same result obtained in the lowenergy effective theory in the mass insertion approximation. In the opposite limit, $\lambda_{\mathrm{IR}} R \gg 1, m^{2}=\left(R / R^{\prime}\right)^{4} / R^{2}$, which is independent of the brane mass term and is always less than the compactification scale $1 / R^{\prime}$.

At distances below the compactification length scale, $q R^{\prime} \gg 1$, but still above the AdS length scale, $q R \ll 1$, the leading term in the propagator is

$$
\Delta\left(R, R, q^{2}\right) \rightarrow \frac{2}{R} \frac{1}{\square} \delta^{(4)}\left(x^{\prime}-x\right) .
$$

\section{Mass on the $\mathrm{UV}$ brane $\left(\boldsymbol{\lambda}_{\mathrm{IR}}=\mathbf{0}, \boldsymbol{\lambda}_{\mathrm{UV}} \neq \mathbf{0}\right)$}

Using the asymptotic properties of the Bessel functions, it is straightforward to perform the long distance limit $q R \ll 1$ while still probing the extra dimension $q R^{\prime} \gg 1$, and we obtain the asymptotic form of the propagator with both legs on the UV brane

$$
\Delta\left(R, R, q^{2}\right) \rightarrow \frac{2}{R} \frac{1}{\square-m^{2}} \delta^{(4)}\left(x^{\prime}-x\right),
$$

where

$$
m^{2}=2 \frac{\lambda_{\mathrm{UV}}}{R},
$$

to leading order in $\lambda_{\mathrm{UV}} R$.

While the validity of this result requires $\lambda_{\mathrm{UV}} R \ll 1$, it does not restrict the relative size between the graviton mass $m$ and the compactification scale $1 / R^{\prime}$. Thus we can 
use these results in the limit that the IR brane is decoupled, $1 / R^{\prime} \rightarrow 0$. In this limit there is a mass gap, with a continuum of bulk graviton states down to zero. Following [25], we expect the massive graviton (A13) to be unstable. To see this one has to compute the propagator for timelike momenta $p^{2}=-q^{2}<0$.

Sending the IR brane to infinity, $R / R^{\prime} \rightarrow 0$, and imposing that positive frequency waves are ingoing at $z=\infty$ (or equivalently, performing the analytic continuation of the propagator in (A5) and (A6)), gives

$$
\Delta\left(z, z^{\prime}\right)=\frac{\left(z z^{\prime}\right)^{2}}{R^{3}} \frac{H_{2}^{(1)}\left(q z_{>}\right) H_{2}^{(1)}\left(q z_{<}\right) \mathcal{B}\left(q z_{>}\right)}{q H_{1}^{(1)}(q R)-\lambda_{\mathrm{UV}} H_{2}^{(1)}(q R)}
$$

with

$$
\begin{aligned}
\mathcal{B}\left(q z_{>}\right)= & q J_{1}(q R)-\lambda_{\mathrm{UV}} J_{2}(q R)-\left[q H_{1}^{(1)}(q R)\right. \\
& \left.-\lambda_{\mathrm{UV}} H_{2}^{(1)}(q R)\right] \frac{J_{2}\left(q z_{<}\right)}{H_{2}^{(1)}\left(q z_{<}\right)} .
\end{aligned}
$$

$H_{\nu}^{(1)}=J_{\nu}+i Y_{\nu}$ is the Hankel function of the first kind of order $\nu$.

As a check, note that in the limit of a vanishing FierzPauli mass, $\lambda_{\mathrm{UV}}=0$, we recover the RS2 propagator found in [30].

The interesting result is the presence of a pole at

$$
q \frac{H_{1}^{(1)}(q R)}{H_{2}^{(1)}(q R)}-\lambda_{\mathrm{UV}}=0 .
$$

This is almost identical to the equation solved by [25] in a related context. There they found a complex pole. Following [25], we expand this equation in the $q R \ll 1$ limit using asympotic properties of the Bessel functions and

$$
\frac{H_{1}^{(1)}(q R)}{H_{2}^{(1)}(q R)}=\frac{Y_{1}(q R)}{Y_{2}(q R)}\left(1-i \frac{J_{1}(q R)}{Y_{1}(q R)}+\cdots\right)
$$

where the ellipses denoted terms suppressed by $q R$. The solution to (A16) is given by

$$
m=m_{0}-i \Gamma
$$

with $m_{0}^{2}=2 \lambda_{\mathrm{UV}} / R$ and $\Gamma / m_{0}=\pi\left(m_{0} R\right)^{2} / 8$.

\section{APPENDIX B: NON-FIERZ-PAULI MASS TERM ON THE IR BRANE}

This appendix analyzes the gravitational spectrum for the case of a generic non-Fierz-Pauli mass term for the graviton on the IR brane.

The bulk action is

$$
S_{\text {bulk }}=\int d^{5} x \sqrt{g}\left(\frac{\mathcal{R}}{2 \kappa_{5}^{2}}+\cdots\right)
$$

where the ... includes, in particular, the GibbonsHawking boundary terms. The action on the IR brane is taken to be

$$
S_{\mathrm{IR}}=\frac{1}{8 \kappa_{5}^{2}}\left(\frac{R}{R^{\prime}}\right)^{3} \int d^{4} x\left(a h_{\mu \nu}^{2}-b h^{2}\right)_{\mid z=R^{\prime}}
$$

The case $a=b=-\kappa_{5}^{2} f_{\mathrm{IR}}^{4} R / R^{\prime}$ gives the Fierz-Pauli mass term studied in Sec. II. Since this brane action is not coordinate invariant, we need to specify the coordinates in which the action has this form. We choose it to describe the so-called GNIR coordinates, where $h_{55}=$ $h_{\mu 5}=0$ locally near the brane.

From the equations of motion we obtain the boundary condition at the IR brane to be

$$
\begin{aligned}
& \left.\left(\partial_{z} h_{\mu \nu}^{\mathrm{GNIR}}-\eta_{\mu \nu} \partial_{z} h^{\mathrm{GNIR}}\right)\right|_{z=R^{\prime}} \\
& \quad=\left.\left(a h_{\mu \nu}^{\mathrm{GNIR}}-b \eta_{\mu \nu} h^{\mathrm{GNIR}}\right)\right|_{z=R^{\prime}}
\end{aligned}
$$

As in four dimensional massive gravity with a non-Fierz-Pauli mass term, here we expect the existence of an additional propagating scalar degree of freedom, corresponding to the trace of the metric.

Indeed, solving the bulk equations of motion and the boundary conditions allows for a nonzero trace of the form

$$
h(x, z)=\Phi(x)+\frac{1}{6}\left(R^{\prime 2}-z^{2}\right) \frac{b-a}{a} \square \Phi(x)
$$

where again $h=h_{\mu}^{\mu}$ and $\square=\partial^{\mu} \partial_{\mu}$ and $\Phi$ is a 4D scalar field. The boundary condition (B3) then simply determines the mass of $\Phi$ :

$$
m_{\Phi}^{2}=\frac{a}{R^{\prime}} \frac{(a-4 b)}{(b-a)} .
$$

Next, we would like to determine whether this field $\Phi$ is a ghost, and whether the radion is still a ghost when the mass term is not of the Fierz-Pauli form. To this end, we will need to compute the off-shell 4D effective action.

First note that on-shell and in GNIR coordinates the most general solution to the bulk equations of motion and the IR boundary condition is given by

$$
\begin{aligned}
h_{\mu \nu}^{\mathrm{GNIR}}= & H_{\mu \nu}(x, z)+\left(-\frac{z^{4}}{2 R^{3}}+\gamma\right) \partial_{\mu} \partial_{\nu} f(x) \\
& +\lambda_{1}(z) \partial_{\mu} \partial_{\nu} \Phi(x)+\lambda_{2} \Phi(x) \eta_{\mu \nu}
\end{aligned}
$$

where the function $\lambda_{1}$ and the two constants $\lambda_{2}$ and $\gamma$ are given by

$$
\begin{gathered}
\gamma=\frac{R^{\prime 4}}{2 R^{3}}-\frac{2}{a} \frac{R^{13}}{R^{3}}, \\
\lambda_{2}=\frac{a-b}{3 a}, \\
\lambda_{1}(z)=\frac{4 b-a-\frac{1}{2}\left(z^{2}-R^{\prime 2}\right)(b-a) m_{\Phi}^{2}}{3 a m_{\Phi}^{2}} .
\end{gathered}
$$

Satisfying the boundary conditions and equations of mo- 
tion implies that (i) $H_{\mu \nu}$ is transverse and traceless, (ii) $f$ is massless and it is identified with the radion of the previous sections, and (iii) $\Phi$ is the additional degree of freedom identified above with mass given by (B5).

To find the effective four dimensional action for these states we need to provide an off-shell decomposition of the metric fluctuation $h_{\mu \nu}$. The decomposition (B6) is unique once $f$ is related to the brane bending of the UV brane in the GNIR coordinates and once $\Phi$ is defined as the trace of the metric fluctuation at the boundary :

$$
\left.h^{\mathrm{GNIR}}\right|_{z=R^{\prime}} \equiv \Phi
$$

This provides for an off-shell definition of the trace of $H_{\mu \nu}$.

The effective action is most easily computed in the coordinate system where the branes are parallel and fixed at $z=R$ and $z=R^{\prime}$ (so-called "rad" coordinates). Thus in the action given below, the metric appearing there is in the rad coordinates. The metric in these coordinates is obtained by transforming from GNIR coordinates to a coordinate system with both branes parallel. This gives

$$
\begin{aligned}
h_{\mu \nu}^{\mathrm{rad}}= & h_{\mu \nu}^{\mathrm{GNIR}}-\frac{2}{R} \eta_{\mu \nu} F(z) \xi(x) \\
& -2 \int_{R}^{z} d z \frac{z^{\prime}}{R} F\left(z^{\prime}\right) \partial_{\mu} \partial_{\nu} \xi(x), \\
h_{55}= & \frac{2 z}{R} F^{\prime} \xi(x)
\end{aligned}
$$

where

$$
\xi(x)=f(x)+\frac{R}{6} \frac{b-a}{a} \Phi(x)
$$

is the transformation needed to straighten the UV brane.

The five dimensional action is given by

$$
\begin{aligned}
S= & -\frac{1}{4 \kappa_{5}^{2}} \int d^{5} x\left(\frac{R}{z}\right)^{3} h^{A B} E_{A B}\left[h_{C D}\right]-\frac{1}{8 \kappa_{5}^{2}} \int d^{4} x\left(\frac{R}{z}\right)^{3} \\
& \times\left.\left[h^{\mu \nu} h_{\mu \nu}^{\prime}-h h^{\prime}\right]\right|_{R} ^{R^{\prime}}+\left.\frac{3}{8 \kappa_{5}^{2}} \int d^{4} x \frac{R^{3}}{z^{4}} h_{55} h\right|_{R} ^{R^{\prime}} .
\end{aligned}
$$

To this must be added the non-FP brane mass term (B2). Each of these terms require some explanation. The variation of the first term gives (5)-(7), the linearized equations of the motion in the bulk. The terms in the second line are the linear equivalent of the Gibbons-Hawking terms: variation of the term on the first line produces terms on the boundary that are canceled by the variation of the terms appearing in the second line. All boundary terms of the type $O\left(\delta h_{A B}^{\prime}\right)$ are canceled this way. Terms that do not cancel are of the form $\delta h^{\mu \nu} \mathcal{O}_{\mu \nu}$. Requiring that they vanish gives the boundary conditions in rad coordinates. Using (B11), one finds they are equivalent to the GNIR boundary conditions (B3) that were previously inferred from the equations of motion.

As previously mentioned, to this action must be added the non-Fierz-Pauli brane action. The only important point to note is that it must be evaluated in GNIR coordinates. (We could evaluate it in rad coordinates, but that would involve a lengthy substitution of $h^{\mathrm{GNIR}}$ in terms of $h^{\text {rad }}$ into the brane action.)

After a lengthy computation, substituting (B11) into the bulk action (B13), using (B6), and including the brane mass term action (B2), gives (without of course using the four dimensional equations of motion)

$$
\begin{aligned}
S_{\text {eff }}= & -\frac{1}{4 \kappa_{5}^{2}} \int_{R}^{R^{\prime}} d^{5} z \frac{R^{3}}{z^{3}} H^{\mu \nu} E_{\mu \nu}\left[H_{\rho \sigma}\right]+\frac{a}{8 \kappa_{5}^{2}} \frac{R^{3}}{R^{\prime 3}} \\
& \times \int d^{4} x\left(H_{\mu \nu}^{2}-H^{2}\right)_{\mid z=R^{\prime}}-\frac{1}{8 \kappa_{5}^{2}} \int d^{4} x \frac{R^{3}}{z^{3}} \\
& \times\left.\left[H^{\mu \nu} H_{\mu \nu}^{\prime}-H H^{\prime}\right]\right|_{R} ^{R^{\prime}}-\frac{3}{2 \kappa_{5}^{2} R} \int d^{4} x f \square f \\
& +\frac{(b-a)^{2} R^{3}}{24 a^{2} \kappa_{5}^{2} R^{\prime 2}} \int d^{4} x \Phi \square \Phi+\frac{(b-a)(4 b-a) R^{3}}{24 a \kappa_{5}^{2} R^{13}} \\
& \times \int d^{4} x \Phi^{2} .
\end{aligned}
$$

The first two lines describe the action for the massive gravitons and their (linearized) Gibbons-Hawking terms. Note that for the massive gravitons their mass term has been written in the Fierz-Pauli form. This guarantees that for these states there are 5 on-shell degrees of freedom. The last term in the second line and all the terms in the last line describe the quadratic action for the radion and the $\Phi$ field.

We briefly highlight many significant cancellations that occured before arriving at this result. First note that both the radion and the $\Phi$ field have decoupled from each other and from all the spin-2 gravitons. This reassures us that at the quadratic level (B6) correctly decouples all the fields from each other. Further, all quadratic terms involving more than two derivatives also canceled.

From the action (B14) we find that $\Phi$ is not a ghost, in contrast to what occurs in purely four dimensional massive gravity with a non-FP mass term. This may not be surprising, since in the AdS/CFT correspondence the nonFP mass term on the IR brane does not correspond in the CFT to adding a non-FP mass term, but rather to breaking general coordinate invariance in the $\mathrm{IR}^{8}{ }^{8}$ From the action (B14), we read off that the mass of $\Phi$ is given by

\footnotetext{
${ }^{8}$ We have explicitly checked that when a non-Fierz-Pauli mass term is added on the UV brane the scalar field $\Phi$ is now a ghost as it could have also been guessed from the AdS/CFT correspondence.
} 


$$
m_{\Phi}^{2}=\frac{a}{R^{\prime}} \frac{(a-4 b)}{(b-a)},
$$

which agrees with the previous computation using the 5D equations of motion. This provides a nontrivial consistency check that the computation of the effective action is correct.

We find that even for the more general non-Fierz-Pauli mass term the radion is still a ghost. The value of its kinetic term is independent of whether or not the brane mass term has the Fierz-Pauli form.
These results generalize our conclusion that the radion is a ghost when the mass term has the Fierz-Pauli form. That is, in a theory with a non-Fierz-Pauli mass term on the IR brane the radion is always a ghost.

Note added.-While this work was being completed, a work appeared [31] that proposes a long distance modification of gravity based on a Lorentz violating theory. The model makes use of a ghost that condenses. A connection between the presence of ghosts and Lorentz violations has also recently been studied in [21].
[1] I. I. Kogan, S. Mouslopoulos, and A. Papazoglou, Phys. Lett. B 501, 140 (2001); I. I. Kogan, S. Mouslopoulos, A. Papazoglou, G. G. Ross, and J. Santiago, Nucl. Phys. B584, 313 (2000); S. Mouslopoulos and A. Papazoglou, J. High Energy Phys. 11 (2000) 018.

[2] R. Gregory, V. A. Rubakov, and S. M. Sibiryakov, Phys. Rev. Lett. 84, 5928 (2000).

[3] C. Csáki, J. Erlich, and T. J. Hollowood, Phys. Rev. Lett. 84, 5932 (2000); C. Csáki, J. Erlich, T. J. Hollowood, and J. Terning, Phys. Rev. D 63, 065019 (2001); G. R. Dvali, G. Gabadadze, and M. Porrati, Phys. Lett. B 484, 112 (2000); Phys. Lett. B 484, 129 (2000).

[4] G. R. Dvali, G. Gabadadze, and M. Porrati, Phys. Lett. B 485, 208 (2000); E. Kiritsis, N. Tetradis, and T. N. Tomaras, J. High Energy Phys. 08 (2001) 012; 03 (2002) 019; G. Dvali, G. Gabadadze, and M. Shifman, Phys. Rev. D 67, 044020 (2003); I. Antoniadis, R. Minasian, and P. Vanhove, Nucl. Phys. B648, 69 (2003); S. L. Dubovsky and V. A. Rubakov, Phys. Rev. D 67, 104014 (2003); M. Kolanovic, M. Porrati, and J.W. Rombouts, Phys. Rev. D 68, 064018 (2003).

[5] G. Gabadadze and A. Gruzinov, hep-th/0312074.

[6] L. Pilo, R. Rattazzi, and A. Zaffaroni, J. High Energy Phys. 07 (2000) 056.

[7] N. Arkani-Hamed, H. Georgi, and M. D. Schwartz, Ann. Phys. (N.Y.) 305, 96 (2003).

[8] T. Damour and I. I. Kogan, Phys. Rev. D 66, 104024 (2002); T. Damour, I. I. Kogan and A. Papazoglou, Phys. Rev. D 66, 104025 (2002); 67, 064009 (2003).

[9] M. A. Luty, M. Porrati, and R. Rattazzi, J. High Energy Phys. 09 (2003) 029.

[10] V. A. Rubakov, hep-th/0303125.

[11] S. L. Dubovsky and M.V. Libanov, J. High Energy Phys. 11 (2003) 038.

[12] M. Fierz and W. Pauli, Proc. R. Soc. London A 173, 211 (1939).

[13] H. van Dam and M. J. Veltman, Nucl. Phys. B22, 397 (1970); V. I. Zakharov, JETP Lett. 12, 312 ( 1970).

[14] A. I. Vainshtein, Phys. Lett. 39B, 393 (1972).
[15] C. Deffayet, G. R. Dvali, G. Gabadadze, and A. I. Vainshtein, Phys. Rev. D 65, 044026 (2002).

[16] A. Gruzinov, astro-ph/0112246.

[17] M. Porrati, Phys. Lett. B 534, 209 (2002).

[18] L. Randall and R. Sundrum, Phys. Rev. Lett. 83, 4690 (1999).

[19] C. Csáki, C. Grojean, H. Murayama, L. Pilo, and J. Terning, Phys. Rev. D 69, 055006 (2004); C. Csáki, C. Grojean, L. Pilo, and J. Terning, Phys. Rev. Lett. 92, 101802 (2004); C. Csáki, C. Grojean, J. Hubisz, Y. Shirman, and J. Terning, hep-ph/0310355.

[20] M. Luty, "Theory and Phenomenology of Physics at the TeV Scale," Aspen Center for Physics, Colorado, USA, July 2nd 2003 (unpublished); N. Arkani-Hamed, "Superstring Cosmology Conference," KITP, Santa Barbara, California, USA, Oct. 24th 2003.

[21] J. M. Cline, S. Jeon, and G. D. Moore, hep-ph/0311312.

[22] D. G. Boulware and S. Deser, Phys. Rev. D 6, 3368 (1972).

[23] Z. Chacko and E. Ponton, J. High Energy Phys. 11 (2003) 024.

[24] J. Garriga and T. Tanaka, Phys. Rev. Lett. 84, 2778 (2000).

[25] S. L. Dubovsky, V. A. Rubakov, and P. G. Tinyakov, Phys. Rev. D 62, 105011 (2000).

[26] E. C. Titchmarsh, Eigenfunction Expansions, Part I (Oxford University, New York, 1962).

[27] C. Charmousis, R. Gregory, and V. A. Rubakov, Phys. Rev. D 62, 067505 (2000).

[28] Z. Chacko and P. J. Fox, Phys. Rev. D 64, 024015 (2001).

[29] N. Arkani-Hamed and M. D. Schwartz, Phys. Rev. D 69, 104001 (2004); M. D. Schwartz, Phys. Rev. D 68, 024029 (2003); C. Deffayet and J. Mourad, Phys. Lett. B 589, 48 (2004).

[30] S. B. Giddings, E. Katz, and L. Randall, J. High Energy Phys. 03 (2000) 023.

[31] N. Arkani-Hamed, H. C. Cheng, M. Luty, and S. Mukohyama, J. High Energy Phys. 05 (2004) 074; N. Arkani-Hamed, P. Creminelli, S. Mukohyama, and M. Zaldarriaga, J. Cosmol. Astropart. Phys. 04 (2004) 001. 